\title{
Numerical Simulation Method for Fracture Effect of Corroded Steel Strand Under Tension
}

\author{
Fumin Li, Yaxu Qu, Yu Zhao, and Rong Lu \\ Jiangsu Key Laboratory of Environmental Impact and Structural Safety in Engineering, \\ China University of Mining and Technology
}

\begin{abstract}
Steel strand presents characteristic pitting corrosion in chloride environment, which leads PC structures to a serious structural failure even with light presentational corrosion. So it is necessary to analyze the fracture effect of steel strand caused by pitting corrosion. The theoretical analyses are difficult for the presence of complicated threedimension pits, so numerical method becomes a feasible choice. A numerical simulation method for fracture effect of corroded steel strand under tension by the ANSYS software is introduced in this paper, which contains mainly four issues such as fracture criterion, constitutive relation, geometrical models, and simplified mechanical models. The Mises strain is fixed as the fracture criterion parameter because the fracture is stemmed from overlarge plastic deformation. A type of three-line constitutive relation model based on experiment is fixed. The geometrical models of corroded wires with one of the two type pits, namely spheroidic pit and anticlastic pit, are both came from the cylinder cut by a surface rotated from a segment of circular arc. A simplified mechanical model is fixed as the analyzing model, which is a cylinder with $50 \mathrm{~mm}$ length and one pit, one end is fixed rigidly and another end is restricted along the longitudinal axis and applied with area load.
\end{abstract}

\section{INTRODUCTION}

Steel reinforcement corrosion in concrete has long been recognized as a global problem that has caused widespread damages to concrete structures ( $\mathrm{Li}$, Yang, \& Melchers, 2008). It normally occurs due to attacks of aggressive agents such as chloride ions from marine environment, deicing salt, or chloride contaminated aggregates. The formation of corrosion products (rust) involves a substantial volume increase, so the expansive stresses are induced around corroded steel bars causing possible cracking and so far spalling of concrete cover, and thus reducing the serviceability of concrete structures (ACI 365.1R, 2002; Melchers \& Li, 2006; Nuernberger, 2008). Moreover, the tension and bond capacity of steel reinforcement degenerate with the corrosion propagation. So the safety of concrete structures should be reduced too, even the ultimate structural collapse occurring (Podolny, 1992). It was ever estimated 30-years ago that corrosion related maintenance and repairs for concrete infrastructure costed around $\$ 100$ billion per annum in the world (Tuutti, 1982).

A review of literature shows that research on steel corrosion in concrete is more focused on reinforced concrete $(\mathrm{RC})$ structures than on prestressed concrete (PC) structures. Whilst the underlying principles of corrosion science are the same for both reinforcing steel and prestressing steel, the differences in mechanical and physical conditions of the steels, in particular the high level of stresses in prestressing steels (strands), their microstructure, their smooth surface and small cross-sectional areas, give rise for the need to examine the corrosion effects separately in PC structures. Research (e.g., Schupack \& Suarez, 1982) has shown that, the effects of corrosion on structural behavior in these two types of structure are different, with PC structures having less cracking but more serious structural failures, as indicated by more cases of structural collapses of PC structures caused primarily by prestressing steel corrosion.

Both experimental results and field experiences (Podolny, 1992; Valiente, 2001) suggest that a large proportion of structural damages in PC structures, in particular collapses, are caused by pitting corrosion of pretesting steels. It is also shown ( $\mathrm{Li}$, Yuan, Jiang, \& Du, 2008; Li, Yuan, Du, \& Ma, 2009) that, due to the pitting corrosion in three dimension, the proportion of brittle fractures of the prestressing strands increases and the ultimate tensile strength and strain of steel strands decrease remarkably as a result of combined stress concentration and reduction in cross-sectional area of steel strands.

Due to the existence of three-dimensional corrosion pits, it is difficult to achieve the theoretical analysis of various effects of steel strand. In this paper it is considered using the finite element software ANSYS to introduce the numerical simulation methods of tension fracture effect of corroded steel strand. 


\section{CORROSION AND FRACTURE CHARACTER OF STEEL STRAND}

Two main factors lead steel strand to a characteristic pitting corrosion in chloride environment, and the pit configuration is mainly in two types as long spheroidicity and saddle, as shown in Figure 1 (Li, Yuan, Wang, \& Jiang, 2008).

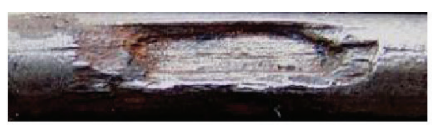

(a)

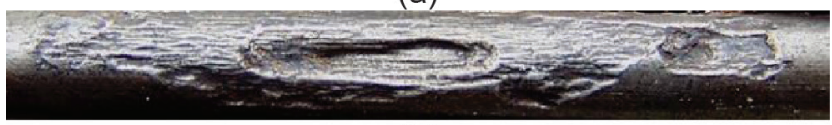

(b)

Figure 1. Spheroidic pit (a) and anticlastic pit (b).

Firstly, the surface of the steel wire of strand is flat and uniform relatively, the passivating film is relatively uniform too. In addition, there are many cementite protective films, which can reduce the area where $\mathrm{Cl}^{-}$can penetrate in, while less corrosive kernels are produced. And the supply of water and oxygen required by corrosion of the kernel is sufficient comparatively; the probability of turning kernel into hole, piece, or pit is higher. It is represented as local and uneven corrosion in the mass.

Secondly, steel strand consists of many drawn pearlite colonies in microstructure. The electrochemical character inside each pearlite colony is similar relatively, but the electrochemical character of each colony is different to others. And then, the corrosion expands only inside certain colony generally. The pearlite colony of the wire represent as long strip or plank parallel to the axes of the wire. When the ferrite inside is corroded, long spheroidic pit or anticlastic pit appears. The uncorroded cementite lamina represents corrosion stripes in the pit.

By tension test, the fracture character of steel strand can be observed ( $\mathrm{Li}$, Yuan, Jiang, \& Du, 2008). The results show that the fractures of all the corroded steel strands include three kinds of basic modes and their combinations, the basic modes are cleavage, fraise and cup; the fractures of the uncorroded steel strands include the latter two kinds of basic modes mentioned above, as well as their combinations. There is no obvious necking near the cleavage fracture, which is macro-brittle fracture; there are necking with different degrees near the cup and fraise fracture, both of which are macro-ductile fracture (Figure 2).

The different macro fracture patterns are corresponded to different fracture mechanisms. According to $\mathrm{Li}$ and Yuan (2007), the fracture source of cleavage fracture is in the edge of pits, where the fracture mechanism includes cleavage fracture and slipping separation fracture. The major fracture mechanism of fracture expansion area is cleavage fracture, and accompanied with dimple fracture; the fracture sources of fraise fracture and cup fracture are in the central area of wire cross-section, and the fracture mechanism is equiaxed dimple fracture. The fracture mechanism of fracture extension area around the wire cross-section (the small ridge for millingcutter fracture, the shear lip for cup-cone fracture) is slipping separation fracture.

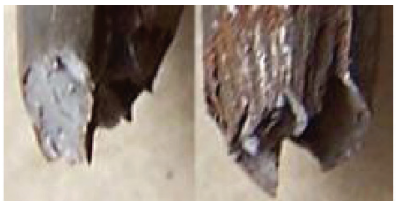

(a)

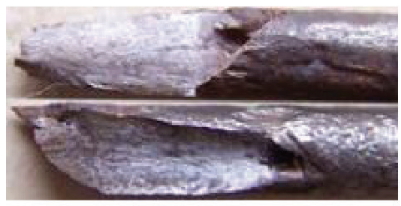

(c)

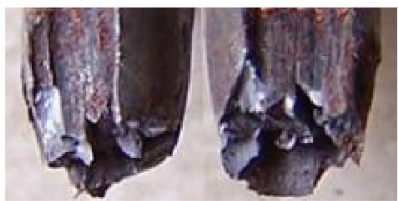

(b)

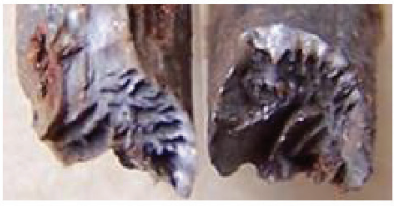

(d)
Figure 2. Fracture modes: (a) cup, (b) fraise, (c) cleavage and (d)-cleavage-fraise.

\section{FRACTURE CRITERION OF CORRODED STEEL STRANDS}

By the test (Li, Yuan, Jiang, \& Du, 2008), we can see that the biggest bearing tensile force of the corroded steel strands appears when the first (batch) wire fractures. Therefore, for a corroded steel strand the first fracture of a wire represents the fracture failure of whole steel strand. So it just needs to discuss the single wire's fracture criterion rather than to discuss the fracture criterion of whole steel strand.

For uncorroded wire of steel strand, when it is subjected to an axis tensile force, the stress state is very simple, so its fracture condition can be directly established based on simple axial tension tests.

But for corroded wire of steel strand, when it is subjected to an axis tensile force, the stress state becomes very complex due to the effect of pitting corrosion. Meanwhile, the presence of corrosion pits may also affect the fracture in the form of wires, which in turn affects the fracture resistance ( $\mathrm{Li} \&$ Yuan, 2007). Thus the fracture condition of corroded steel strand is unable to be established only by simple axis tensile test, the fracture criterion of corroded steel strand is also need to discuss according to the fracture mechanism. 
The macroscopic brittle fracture originated in the crack caused by the local plastic deformation at the bottom of a pit, and once crack occurred it quickly extended to fracture of the whole cross section. That is to say, the initial crack and ultimate fracture occurred almost simultaneously. For macroscopic ductile fracture, the process is: forming plastic deformation near the pit $\rightarrow$ redistributing the stress and strain $\rightarrow$ forming plastic deformation at all section near the zone along the length of pit $\rightarrow$ necking $\rightarrow$ occurring triaxial tensile stress at the center of section $\rightarrow$ fracturing in dimple-type at the center of section $\rightarrow$ occurring separate-slip crack around the wire to form a cuttertype or cup-type fracture. Obviously, the macroductile fracture originates in the plastic deformation which first appeared at the pits, then a more adequate redistribution of stress and strain occurs at each section of wire pits. But the redistribution of stress and strain just occurs in the section and hardly exists between the wires, thus its protective effects of stress relaxation is relatively limited, under these conditions the load increment required to break the whole cross-section will be very limited. Therefore, for macroscopic ductile fracture, there are some little differences between the initial break and ultimate fracture. In order to simplify the problem, once initial fracture effect reaches limit value it can be approximated the overall fracture.

In summary, whether the ductile or brittle fracture in macro, the fracture mechanism in microcosmic is derived from overlarge plastic deformation, thus plastic failure criteria in material mechanics can be used. The theory of specific potential energy of shape change set the beginning yield as the failure criteria, which is rigorous for analyzing the fracture of the corroded wires whose strain gradient is relatively large. In addition, the Mises strain, a strain invariant, is used in plastic theory in common. Therefore, it is set as the fracture criterion here that when the Mises strain reaches the limit, namely

$$
\varepsilon_{\mathrm{v}}=\varepsilon_{\mathrm{v}, \mathrm{u}}
$$

\section{NUMERICAL SIMULATION METHOD}

\subsection{Material property and constitutive relation}

With a typical tensile pearlite microstructure, the wires of steel strand are anisotropic in mechanical properties (and close to orthotropic). To reflect the characteristics of anisotropic accurately, relevant mechanical tests such as the basic two axis (the vertical axis and the horizontal axis of the wire) tensile tests are necessary to be carried out. As is well known, the tensile test along the longitudinal axis is easy to be implemented, but it is difficult to achieve the test along the horizontal axis. While fortunately, the wire breakage performance depends mainly on the performance of the longitudinal axis, so it can be assumed that steel strand is an isotropic material, and all of the constitutive relations along each direction are same as that of the longitudinal axis. Then the ANSYS material model used in the analysis can be assumed to be an isotropic hardening rate uncorrelated plasticity model.

The simplified constitutive relation is determined by the tensile test. The tensile test was carried out with four steel strands of $\phi^{\text {s } 12.7}(1 \times 7) 1860$ and one center wire from the same type steel strand, and their stressstrain curves can be seen in Figure $3(\mathrm{Li}, 2008)$.

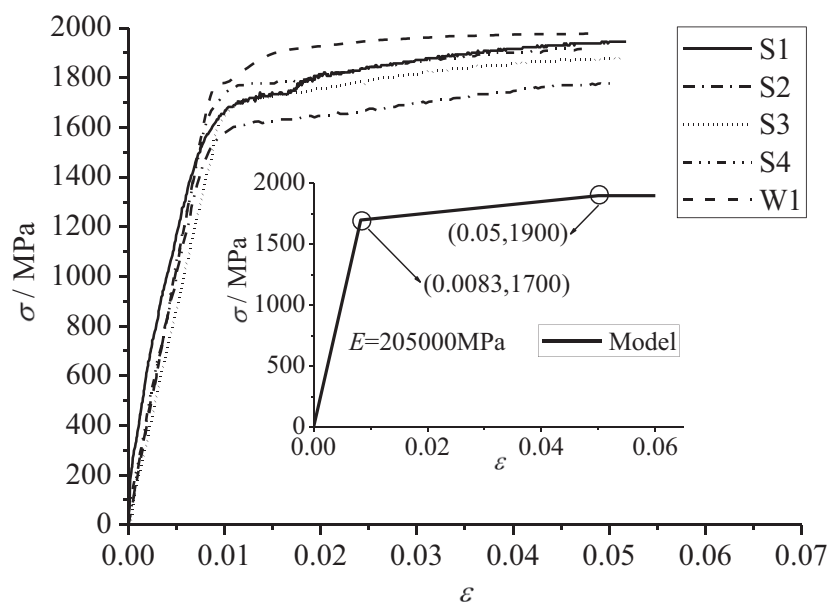

Figure 3. Model of stress-strain of steel strands and the core steel wires.

To analyze each curve comprehensively and for adapting to each isotropic hardening rate uncorrelated plasticity model mentioned above, the Model curve in Figure 3 is intended to use as a representative constitutive relation curve of the strand wire. The curve consists of three segments straight lines. The segment one is elastic stage; the slope of the curve is obtained from the slope of the elastic stage of the core steel wires curve, and the end-point stress values were obtained from the mean yield stress of five curves. The segment two is elastic-plastic reinforced stage; the end-point stress and strain values were both obtained from the average yield stress of five curves. The segment tree is pure plastic stage; it is added only for adapting to the corresponding ANSYS constitutive model.

\subsection{Geometrical models of corroded steel strand}

The steel strand is twisted by some wires and each outside steel wire is spiral around the longitudinal axis. Each outside steel wire clung to one another when the steel strand is tensioned. At the same time, the outside still wires and the core steel wires form a whole and deform consistently. Therefore, the stress state of each outside steel wire is close 
to the state of a straight steel wire and different from the state of an independent spiral wire, where there are geometric large deformation and torque in the straightening process of steel wires. Based on the above consideration, the geometrical model of twisted steel strand can be simplified into the corresponding geometrical model of parallel straight wire unit.

As mentioned above, the geometrical shapes of the corrosion pits in the pre-stressed steel strand wire can be classified into spheroidic and saddle shapes. The simulation processes in the ANSYS of each corrosion pit are described separately below.

The surface rotated by circular arc cuts the steel cylinder and the pit is deleted to obtain the spheroidic corrosion pit, as shown in Figure 4. It is easier to control the depth of pit which is determined by the bottom vertex of rotating surface. The width and length of pit are, respectively, controlled by the curvature and turning radius of the arc, and the specific target values need to be approximated to the actual values by many times trials.

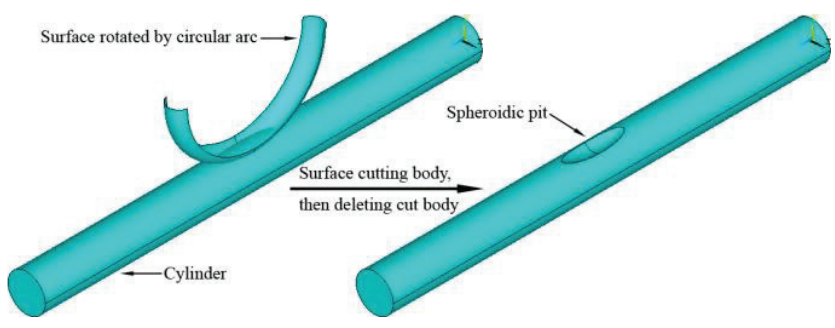

Figure 4. ANSYS model of spheroidic pit.

The method to measure the width of pit is as below. Firstly, a cross section through the bottom vertex of pit should be built. And then, the intersecting line (as arc) of the cross section and the upper surface of pit can be obtained. Lastly, the length of the intersecting line can be calculated by LSUM and the calculated value is the width of pit.

The measuring method of the length is similar to the method of the width. Firstly, a vertical longitudinal section through the bottom vertex of pit should be built. And then, the intersecting line (as the plain wire of cylinder surface) of the longitudinal section and the upper surface of pit can be obtained. Lastly, the length of the intersecting line can be calculated by LSUM and the calculated value is the length of pit.

It is important to be noted that the model of the spheroidic corrosion pit includes the situation that the specific value of the length and width is less than or equal to 1.

The methods to obtain the saddle-shaped corrosion pit and measure the size of pit are similar to the ones of the spheroidic pit. As mentioned above, the surface rotated by circular arc cuts the steel cylinder and the pit is deleted to obtain the corrosion pit. The only difference is that the direction of curvature of the circular arc of saddle-shaped pit is opposite to the spheroidic one, as shown in Figure 5.

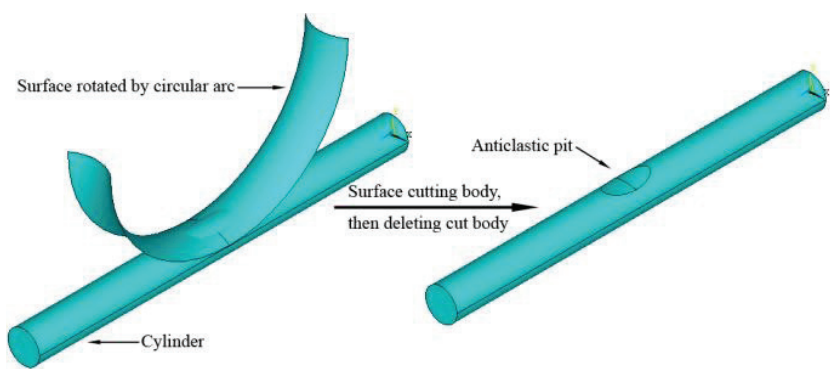

Figure 5. ANSYS model of anticlastic pit.

According to the test observation (Li, Yuan, Wang, \& Jiang, 2008), the pitting corrosion morphologies of steel wires are very typical. An obvious corrosion pit often exists in isolation along the cross section of a steel wire, that is to say, two obvious corrosion pits hardly exist in the same cross section at the same time. In addition, the distribution of corrosion pits along the longitudinal axis of a steel wire is relatively scattered, so the difference of two adjacent corrosion pits is relatively small. Therefore, the geometrical model can be simplified as one corrosion pit in one steel wire by ANSYS to analyze the mechanical effect of one certain corrosion pit.

\subsection{Redistribution of internal force among wires of corroded steel strands and the simplified mechanical model}

The total elongations of each steel wire are same in the tension process of steel strands. At the same time, the friction effect between steel wires is relatively small, so the tension of every cross section in each steel wire is same. Therefore, if one steel wire has local plastic deformation firstly, the wire will appear stress relaxation along the whole length and the local plastic deformation can be relieved. The redistribution of internal force will emerge between steel wires for the steel strand. Obviously, the redistribution of internal force becomes clearer with the shorter tensile specimen. And when the tensile specimen is enough long, the redistribution of internal force can be ignored.

To study the effect of the length of specimen $(I)$ on the redistribution of internal force, three ANSYS models have been built respectively. To simplify the analysis, the most obvious extreme case of the redistribution of internal force is considered, assuming that one corrosion pit on one steel wire of a steel strand. 
The first model is one single cylinder with one corrosion pit whose length is $50 \mathrm{~mm}$. One side of the cylinder is fixed and the other side is coupled along the free degree of vertical axis of the cylinder in order to simulate the rotational displacement around the horizontal axis which is restricted by steel wires in the distance and other wires around. By applying distributed even area loads on the free end, the case that one single corroded steel wire tensioned is simulated, as shown in Figure 6 (recorded as Model 1). The model is a basic model without the redistribution of internal force between the corroded steel wire and other wires.

The second model is a cylinder with a corrosion pit and a parallel straight bar with constant section whose length are both $50 \mathrm{~mm}$. All the free degree of one side are both coupled and fixed. The other sides are both coupled along the free degree of vertical axis and concentrated tensions along vertical axis are applied on the end. The cylinder represents the corroded steel wire and the straight bar represents other uncorroded steel wires, as shown in Figure 6(b). The model (recorded as Model 2) can be used to simulate the tensile case of a corroded steel wire in a $50-\mathrm{mm}$ steel strand in order to study the redistribution level of internal force between the corroded steel wire and other wires.

The third model is a cylinder with a corrosion pit (50 $\mathrm{mm}$ ) and a coaxial line straight bar with constant section $(550 \mathrm{~mm})$ and a parallel straight bar with constant section $(600 \mathrm{~mm})$. The cylinder and the coaxial line straight bar are linked end to end (to save machine-hour) and coupled along the free degree of vertical axis in order to represent a corroded steel wire (600 mm length). The same ends of the cylinder and the parallel straight bar are coupled and fixed along all free degrees. The same ends of the coaxial line straight bar and the parallel straight bar are coupled along the free degree of vertical axis and concentrated tensions along vertical axis are applied on the end. The parallel straight bar represents other un-corroded steel wires, as shown in Figure 6(c). The model (recorded as Model 3) can be used to simulate the tensile case of a corroded steel wire in a $600 \mathrm{~mm}$ steel strand in order to study the redistribution level of internal force between the corroded steel wire and other wires under static tensile test conditions. The space between tensile chucks can be obtained as $600 \mathrm{~mm}$ because that the scale designation of steel strands is $500 \mathrm{~mm}$ in static tensile tests and work spaces on both ends are allowed.

The simulated steel strand is $\phi^{\mathrm{s}} 12.7(1 \times 7) 1860$. According to the test results, the outside and inside diameter of wire is, respectively, 4.25 and $4.38 \mathrm{~mm}$, so the cross-sectional area of the steel strand can be calculated as $100.2 \mathrm{~mm}^{2}$.

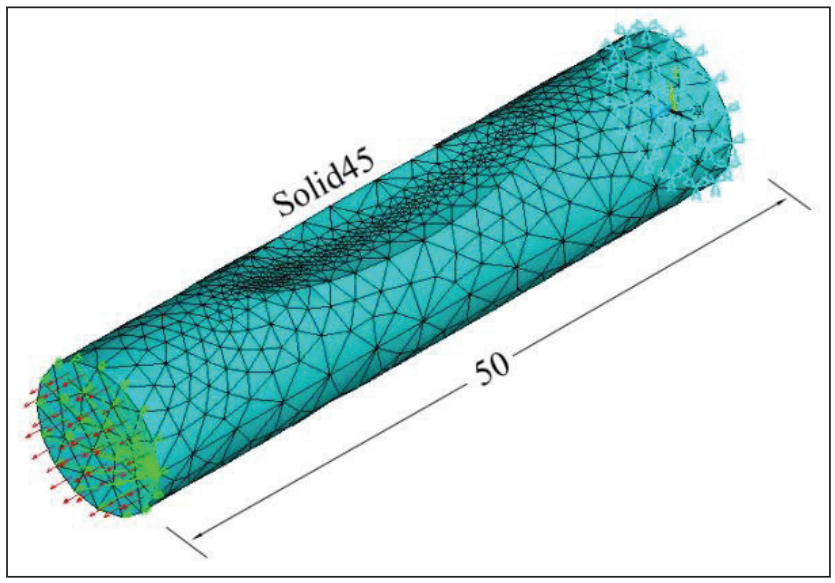

(a)

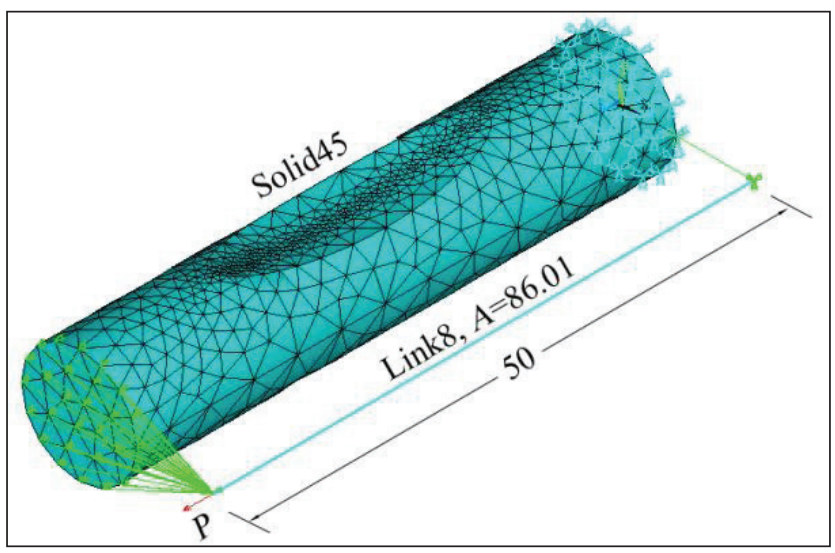

(b)

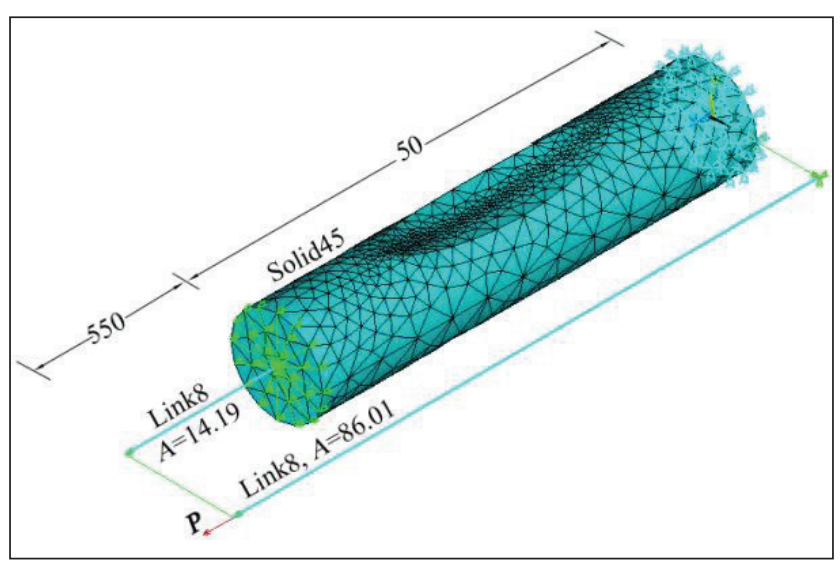

(c)

Figure 6. ANSYS models: (a) Model 1, (b) Model 2, and (c) Model 3.

The Solid45 elements are used to simulate the cylinder and the Link8 3D bar elements are used to simulate the straight bar in ANSYS analysis. The real constant of sectional area is defined as $A=14.19$ in the element Link8 in the coaxial line straight bar. And that is defined as $A=86.01$ in the element Link8 in the parallel straight bar. In nonlinear elastic material models, isotropic enhancement ratio is uncorrelated. And that plasticity models are applied to the material 
models of the two elements. The curve Model represents the constitutive relation of material, as shown in Figure 3. Free tetrahedron grids are divided in the cylinder and the grids increase around the bottom vertex of pyramid pit. Linear grids are divided in the straight bar.

Three above finite element models are applied to analyze the two steel wires that represent different corrosion pit models and the equivalent strain is as shown in Table 1 (Li, 2008) (the representativeness reflects in large corrosion pit and large broken equivalent strain). The redistribution level of internal force can be studied between the corroded steel wire and other wires in two conditions by comparing the results of Model 1 and Model $2 \& 3$.

Table 1. Effects of length of corroded steel strands on redistribution degree of internal force.

\begin{tabular}{lcccc}
\hline Samples & Pits mode & \multicolumn{3}{c}{ Mises strain } \\
\cline { 3 - 5 } number & & $\begin{array}{c}\text { Model } \\
\text { one }\end{array}$ & $\begin{array}{c}\text { Model } \\
\text { two }\end{array}$ & $\begin{array}{c}\text { Model } \\
\text { three }\end{array}$ \\
\hline Sample 1 & Spheroidicity & 0.0649 & 0.0409 & 0.0658 \\
Sample 2 & Saddle & 0.0860 & 0.0478 & 0.0857 \\
\hline
\end{tabular}

It is indicated in Table 1 that the equivalent strain obtained by Model 2 is obvious less than that by Model 1 which is nearly equal to that by Model 3 in every corrosion pit models.

It illustrates that the redistribution level of internal force is relatively high between the corroded steel wire and other wires when the specimens are short. While the length of specimens is $600 \mathrm{~mm}$ in the static tensile test, the redistribution level of internal force is almost 0 . Therefore, the results are almost same by applying Model 1 instead of Model 3.

Therefore, the Model 1 can be applied to simulate corroded steel strands to obtain fracture parameters in tension by the ANSYS.

\section{CONCLUSIONS}

Steel strand presents characteristic pitting corrosion in chloride environment, and the pit configuration is mainly in two types as long spheroidicity and saddle.

The fractures of corroded steel strands include three kinds of basic modes, namely cleavage, fraise and cup, as well as their combinations.

The microcosmic fracture mechanism is almost due to transfinite plastic deformation whether there is macroscopic ductile fracture or brittle fracture. Therefore, the strain invariant (the Mises strain) in plastic theory can be used to be regarded as the criterion parameter when the steel strand breaks in tension.
The steel wires are simplified as isotropic materials because fracture properties of steel wires depend on properties of longitudinal axis in spite of anisotropic characteristics of steel wires. And constitutive relation of every direction is the same as that of longitudinal axis in tension. In nonlinear elastic material models, isotropic enhancement ratio is uncorrelated. And that plasticity models are applied to the material models in ANSYS. The constitutive relation model is simplified by three-line model based on tensile tests.

The surface rotated by circular arc cuts the steel cylinder and the pit is deleted to obtain the spheroidic and saddle-shaped corrosion pits. The only difference is that both the direction of curvature of the circular arc is opposite.

A simplified mechanical model is adopted as the basic model of simulation analysis through trials and compare of simplified and non-simplified mechanical models. The above basic model is one single cylinder with one corrosion pit whose length is $50 \mathrm{~mm}$. One side of the cylinder is fixed and the other side is coupled along the free degree of vertical axis of the cylinder where distributed even area loads are applied.

\section{ACKNOWLEDGMENTS}

Financial support from the National Science Foundation of China (NSFC) with Grant No. 51178454, the Science Foundation of Jiangsu Province, China with Grant No. BK2011222 and the Science Foundation of China University of Mining and Technology with Grant No. 2011QNB19 is gratefully acknowledged.

\section{REFERENCES}

ACI 365.1R. (2002). Service life prediction-state-ofthe-art report. ACI Manual of Concrete PracticePart 5, Farmington Hills.

Li, C. Q., Yang, Y., \& Melchers, R. E. (2008). Prediction of reinforcement corrosion in concrete and its effects on concrete cracking and strength reduction. ACI Materials Journal, 105(1), 3-10.

Li, F. M. (2008). Effect of steel strands corrosion on prestressed concrete structures under chloride environment (Doctor Dissertation), China University of Mining and Technology, Beijing (in Chinese).

Li, F. M., \& Yuan, Y. S. (2007) Fracture behaviour of corroded steel strands under static tension[J]. Journal of Southeast University (nature science edition), 37(5), 904-909. (in Chinese).

Li, F. M., Yuan, Y. S., Du, J. M., \& Ma, H. (2009) Deterioration of tensile behavior of steel strands corroded by chloride. Journal of Southeast University: Natural Science Edition, 39(2), 340-344 (in Chinese) 
Li, F. M., Yuan, Y. S., Jiang, J. H., \& Du, J. M. (2008) Fracture behavior of steel strands corroded in concrete contaminated with salt. Proceeding of the International Conference on Durability of Concrete Structures, Hangzhou China (pp. 669-675).

Li, F. M., Yuan, Y. S., Wang, B., \& Jiang, J. H. (2008) Corrosion characteristics of prestressing steel strands in concrete corroded by chloride attack. Proceeding of the 2nd International Workshop on Life Cycle Management of Coastal Concrete Structures, Hangzhou, China (pp. 95-98).

Melchers, R. E., \& Li, C. Q. (2006). Phenomenological modeling of reinforcement corrosion in marine environments. ACl Materials Journal, 103 (1), 25-32.

Nuernberger, U. (2008). Reasons and prevention of corrosion induced failures of prestressing steel in concrete. Proceeding of the International Conference on Durability of Concrete Structures, Hangzhou China (pp. 137-145).

Podolny, W. (1992) Corrosion of prestressing steels and its mitigation. PCl Journal, 37(5), 34-55.

Schupack, M., \& Suarez, M. G. (1982) Some recent corrosion embrittlement failures of prestressing systems in the United States. PCI Journal, 27(2), 38-55.

Tuutti, K. (1982) Corrosion of steel in concrete. Swedish Cement and Concrete Research Institute, pp. 17-21.

Valiente, A. (2001) Stress corrosion failure of large diameter pressure pipelines of prestressed concrete. Engineering Failure Analysis, 8, 245-261. 